Supporting Information

\title{
The Catalytic M-center of Copper Monooxygenases probed by Rational Design. Effects of Selenomethionine and Histidine Substitution on Structure and Reactivity.
}

Katherine B. Alwan ${ }^{\dagger}$, Evan F. Welch ${ }^{\dagger}$ and Ninian J. Blackburn ${ }^{*}$

tDepartment of Chemical Physiology and Biochemistry, Oregon Health \& Sciences University, Portland, OR 97239

* Corresponding Author blackbni@ohsu.edu Phone 503-346-3428 

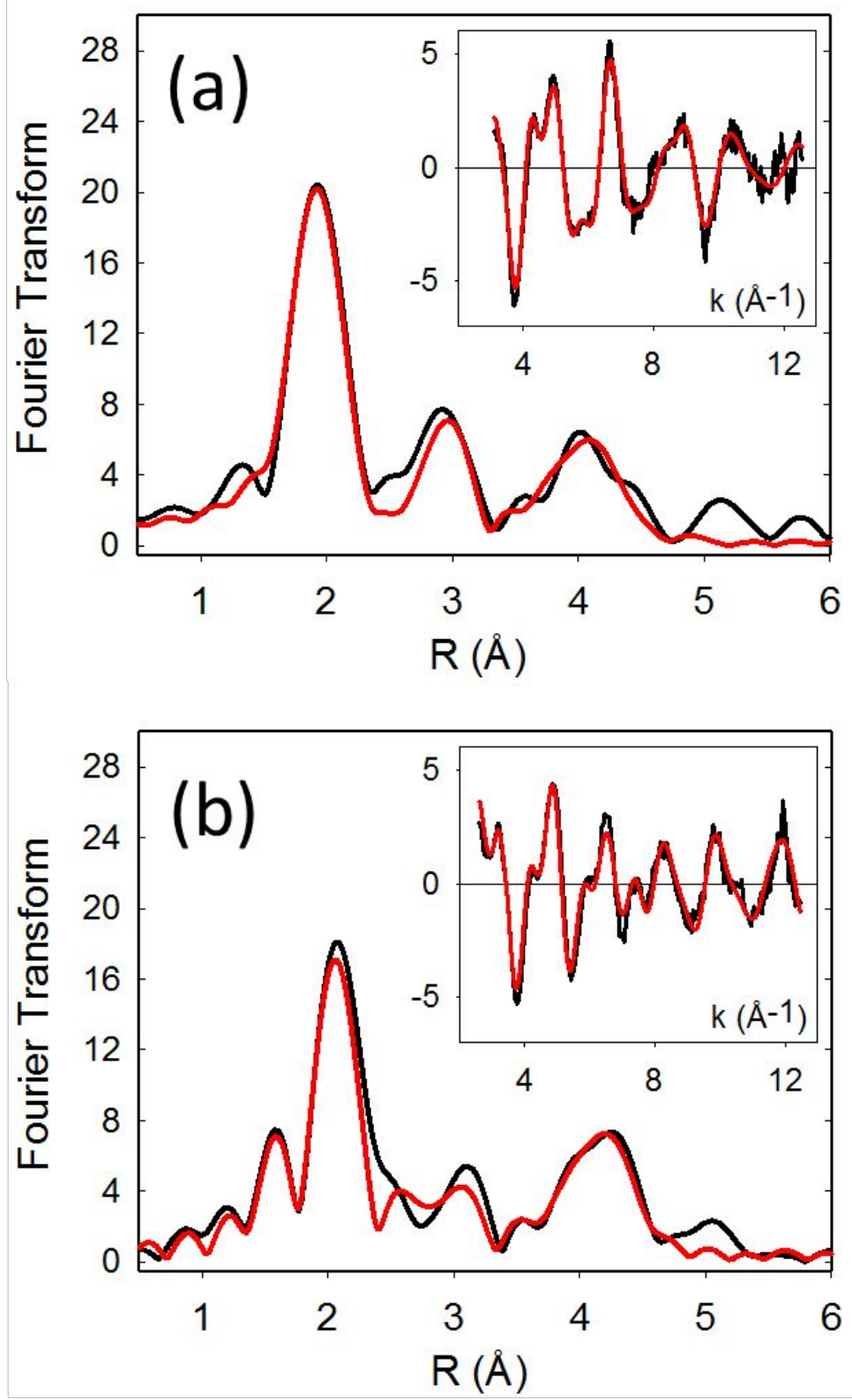

Figure S1. XAS structural characterization of the $\mathrm{Cu}(\mathrm{I})$ forms of the $3 \mathrm{His}(\mathrm{M} 47 \mathrm{H} M 49 \mathrm{H})$ CusF $\mathrm{M}$ site model. Fourier transforms and EXAFS (insets) of (a) unligated and (b) CO-bound complexes. Parameters used in the fits are listed in Table S1. 

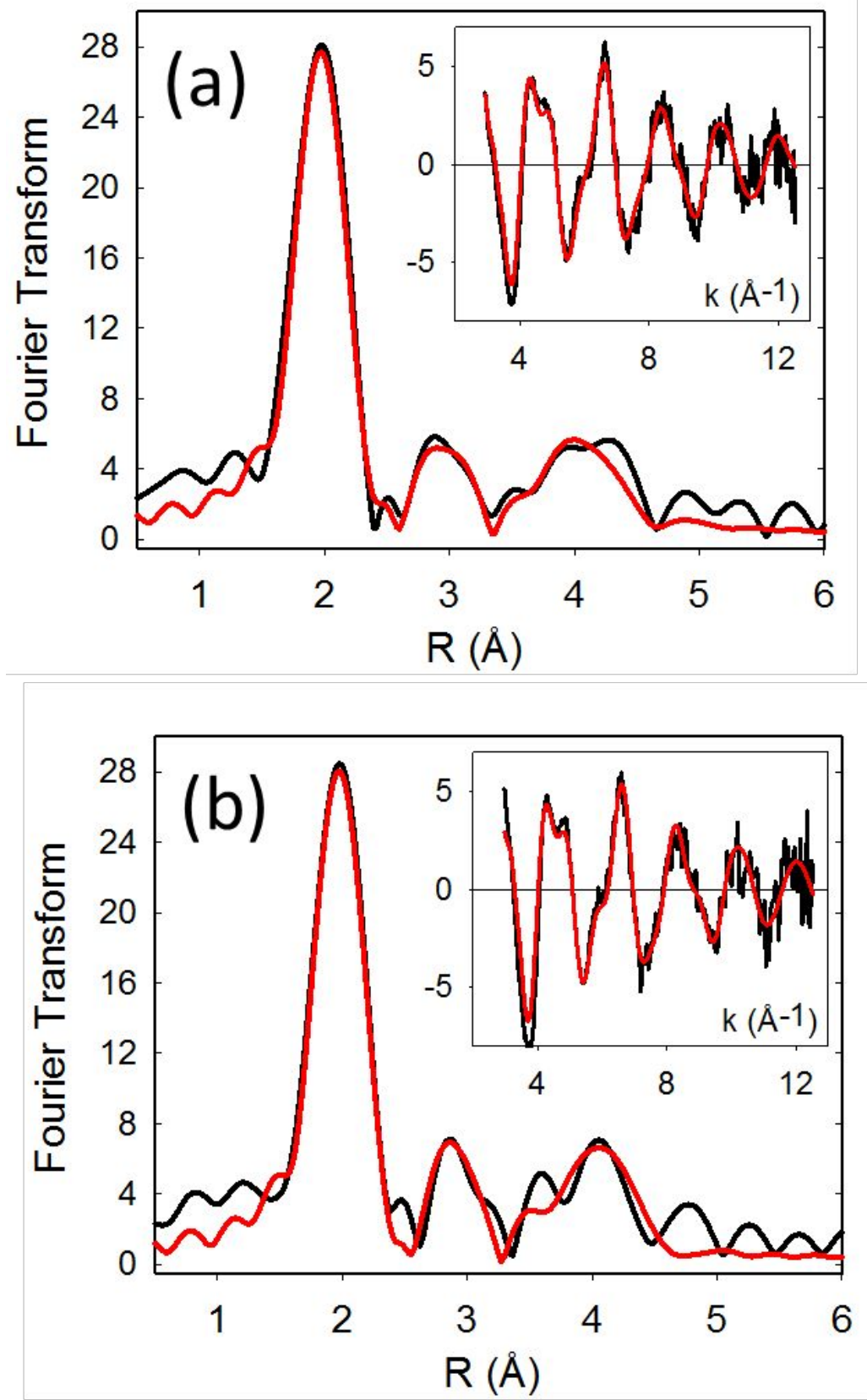

Figure S2. XAS structural characterization of the $\mathrm{Cu}(\mathrm{II})$ forms of the $3 \mathrm{His}(\mathrm{M} 47 \mathrm{HM} 49 \mathrm{H})$ CusF $\mathrm{M}$ site model. Fourier transforms and EXAFS (insets) of (a) unligated and (b) azido-bound complexes. Parameters used in the fits are listed in Table S1. 

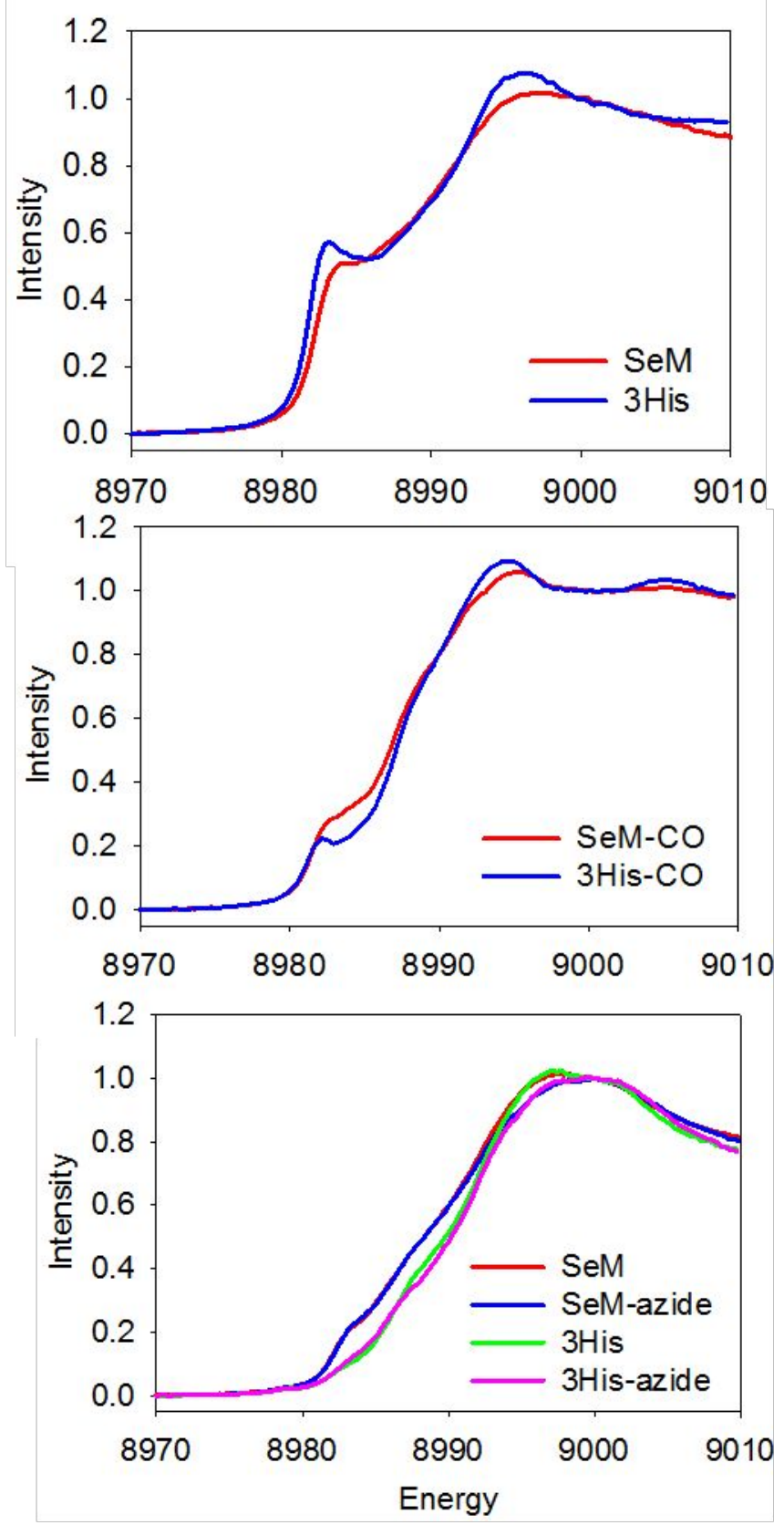

Figure S3. Absorption edges of $\mathrm{M}$-site CusF derivatives. Unligated $\mathrm{Cu}(\mathrm{I})$ (top), $\mathrm{CO}$ complexes (middle) and unligated and azido Cu(II) complexes (bottom). Spectra are color coded as per the legend to each panel. 


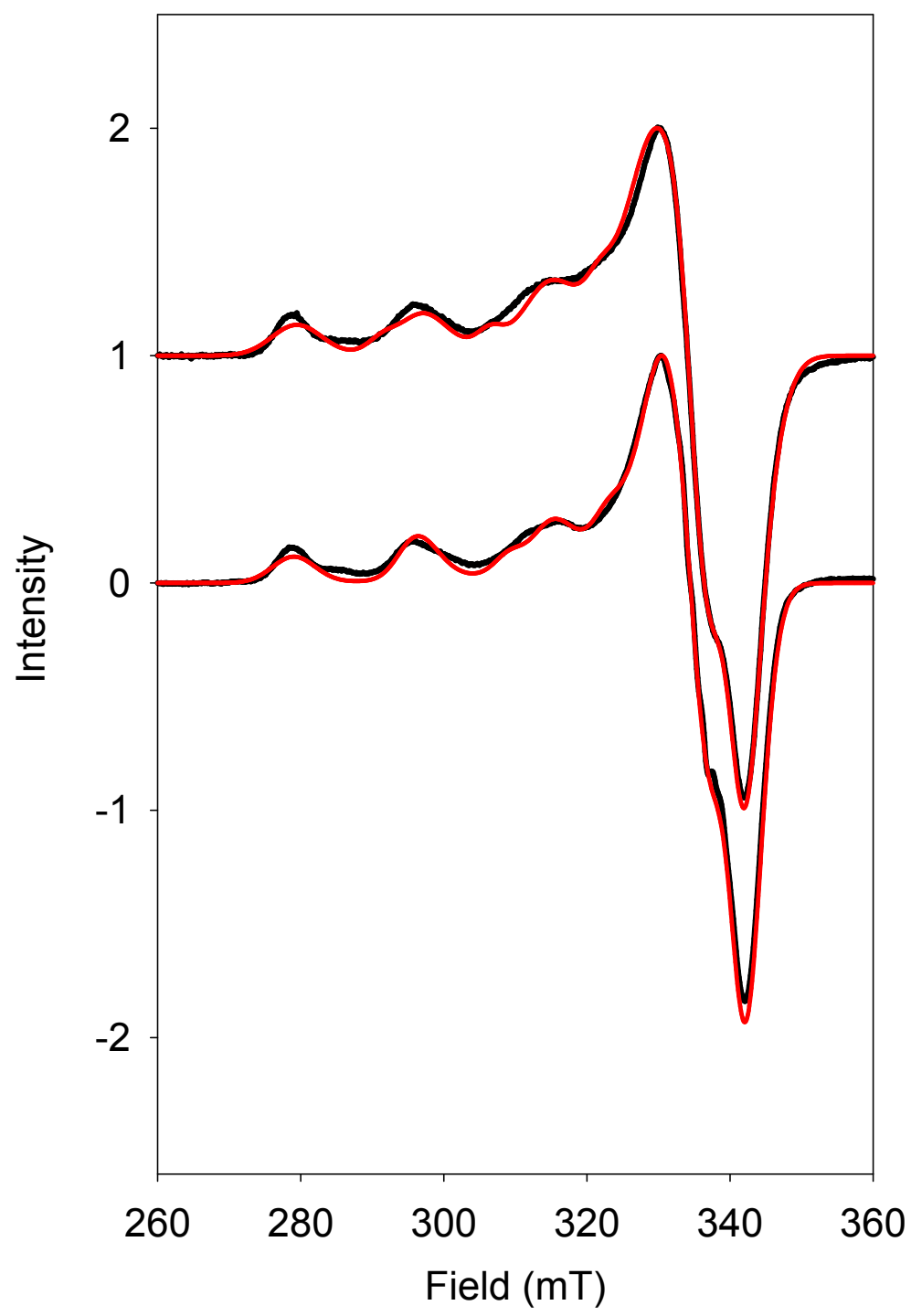

Figure S4. X-band CW EPR spectra of the oxidized SeM-M49H PHM M-site model (bottom trace) and the $\mathrm{M} 47 \mathrm{HM} 49 \mathrm{H}$ (3His) derivative (top trace) determined at pH 5.5. Black traces are experimental data while red traces are simulations using EASYSPIN. The SeM model was simulated by two components in a ratio of $1: 0.3$ with $g$ and $A$ values for component 1 : $\mathrm{gx}=2.065, \mathrm{gy}=2.080, \mathrm{gz}=2.259, \mathrm{Az}=559 \mathrm{MHz}$; and for component 2: $\mathrm{gx}=2.053, \mathrm{gy}=2.065$, $\mathrm{gz}=2.188, \mathrm{Az}=415 \mathrm{MHz}$. The $3 \mathrm{His}$ species also exhibited a two component fit in the ratio 1: 0.45 with $\mathrm{g}$ and $\mathrm{A}$ values for component $1: \mathrm{gx}=2.054, \mathrm{gy}=2.073, \mathrm{gz}=2.271, \mathrm{Az}=483 \mathrm{MHz}$; and for component 2: $\mathrm{gx}=2.024, \mathrm{gy}=2.121, \mathrm{gz}=2.281, \mathrm{Az}=395 \mathrm{MHz}$. Spectra were collected at a temperature of $100 \mathrm{~K}$, microwave frequency $9.678 \mathrm{GHz}, 100 \mathrm{KHz}$ modulation, $10 \mathrm{G}$ modulation amplitude, $20 \mathrm{~mW}$ microwave power and $1000 \mathrm{G}$ sweep width with the field centered at 3100 G. 

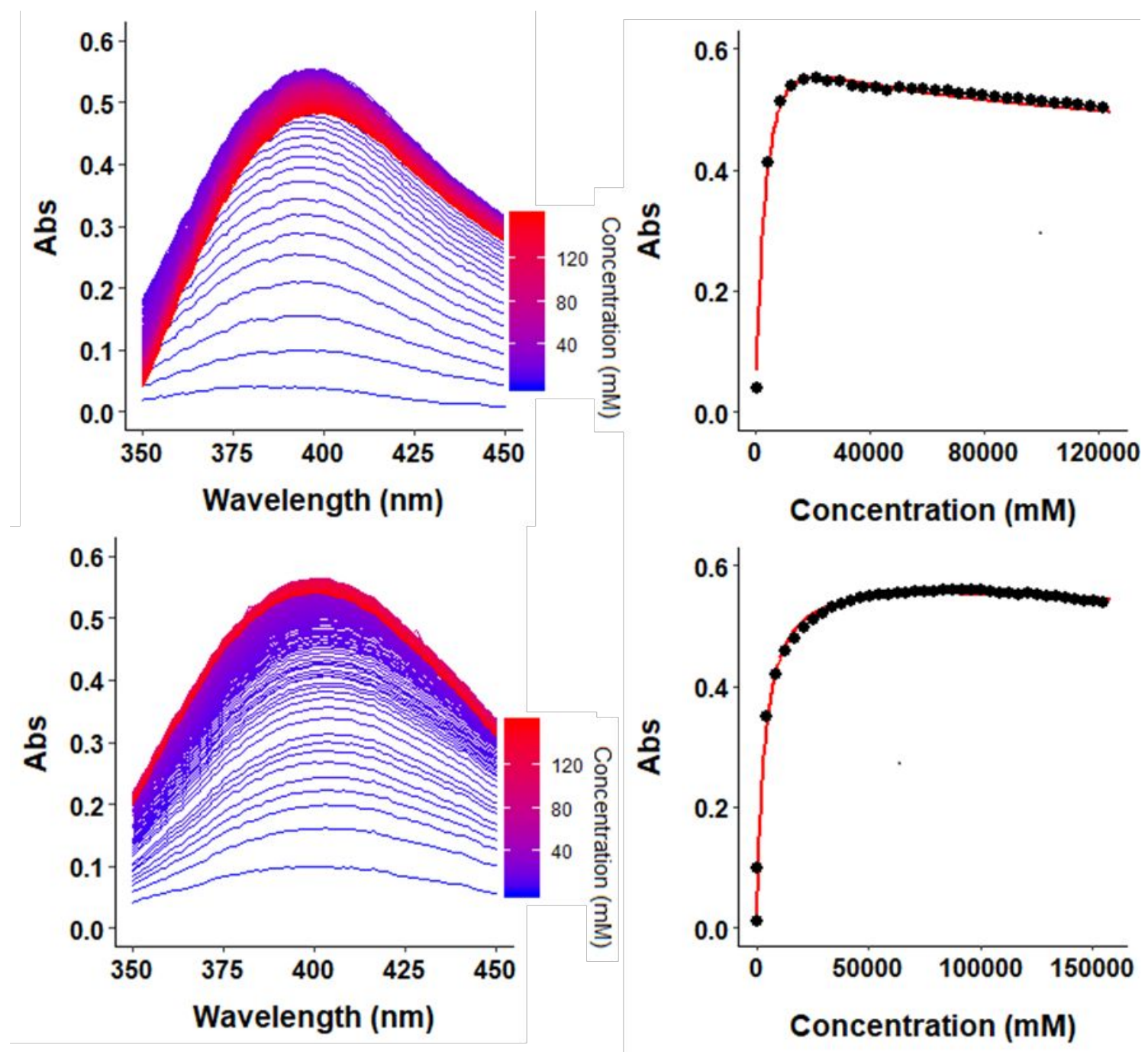

Figure S5. Azide titrations of the oxidized CusF M-site model variants via additions of aliquots of $2 \mathrm{M}$ sodium azide to a final concentration of $100 \mathrm{mM}$. The resulting absorbance at $390 \mathrm{~nm}$ was fit to a formation curve as described in the text. $K_{D}$ values extracted from the data are listed in Table 3 of the main manuscript. Top panels are for the Sem-M49H and bottom panels are for the $\mathrm{M} 4749 \mathrm{H}$ (3His) azido complexes. 

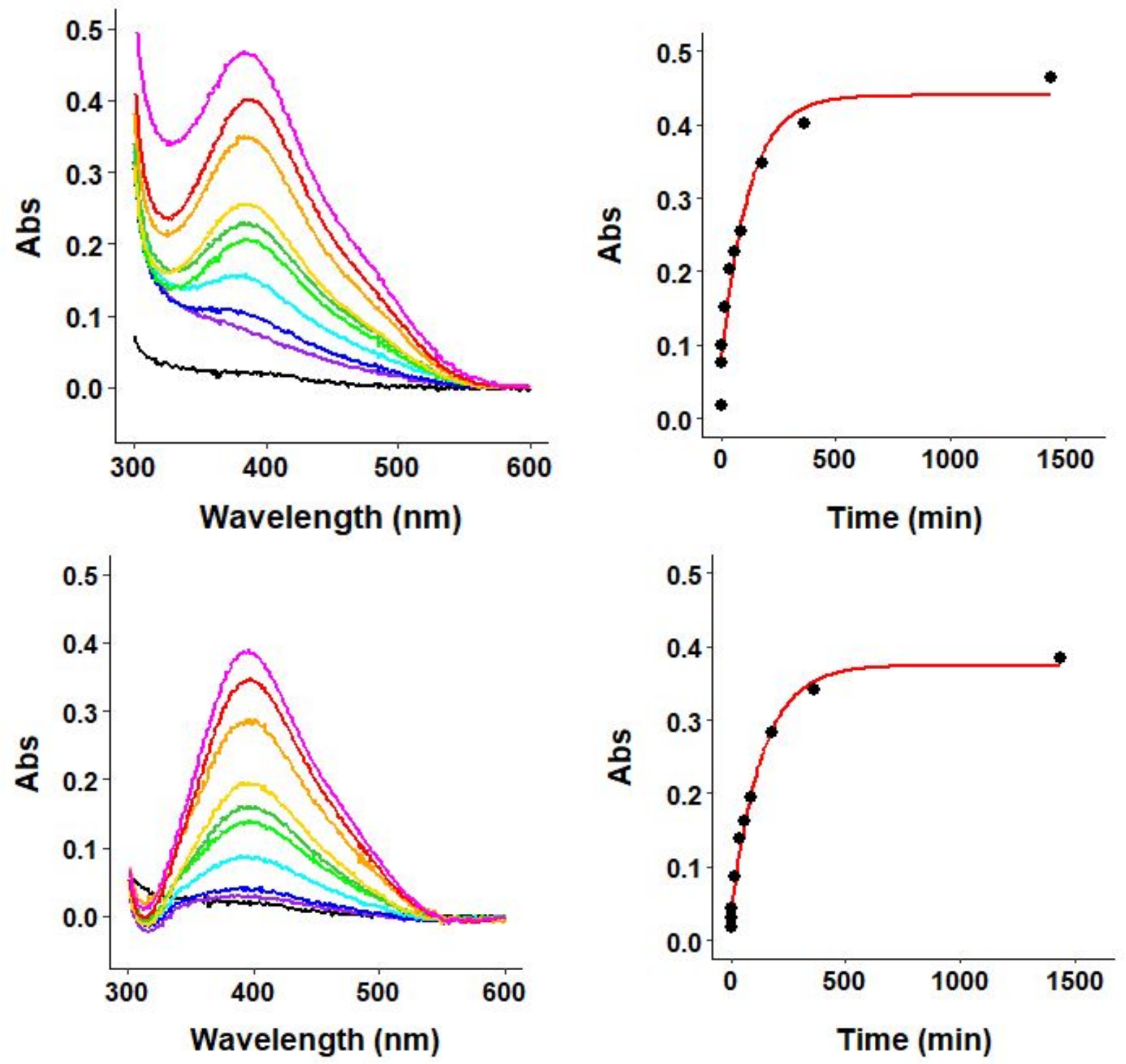

Figure S6. Oxidation kinetics of reduced CusF M-site model variants measured by the addition of $100 \mathrm{mM}$ sodium azide and monitored by formation of the $\mathrm{Cu}(\mathrm{II})$ azido complex at $400 \mathrm{~nm}$. The reaction was sampled at 0min (black), $1 \mathrm{~min}$ (purple), $5 \mathrm{~min}$ (blue), 15min (cyan), 40min (green), 60min (dark green), 90min (yellow), 180min (gold), 360min (red) and 1440min (pink). The resulting absorbance at $400 \mathrm{~nm}$ was plotted versus time fit to a single exponential rise to maximum with rate constants as listed in Table 3 of the main manuscript. Top panels are for the SeM-M49H and bottom panels are for the $\mathrm{M} 4749 \mathrm{H}(3 \mathrm{His})$ azido complexes. 

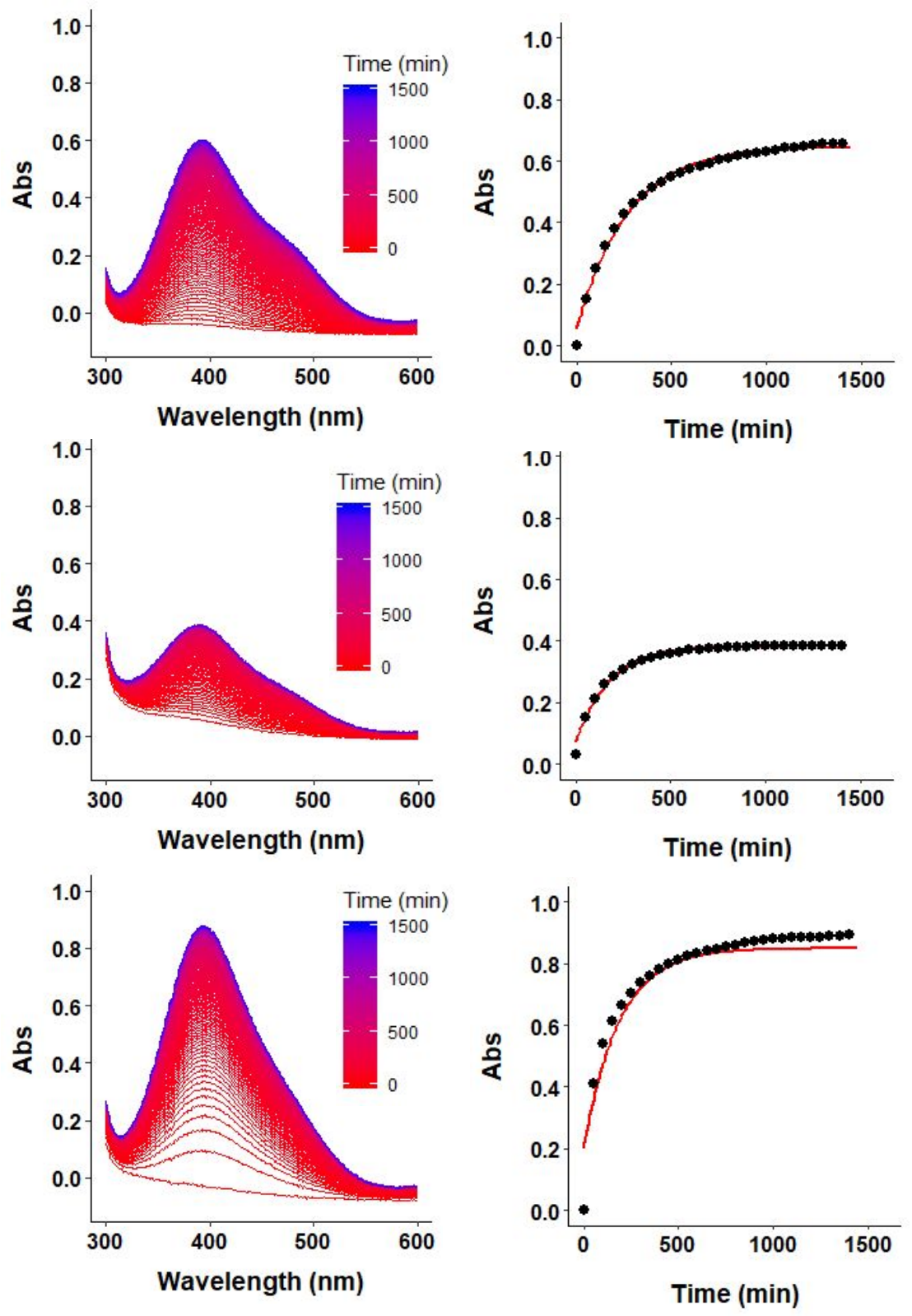

Figure S7. Oxidation kinetics of the reduced CusF M-site model variants measured in the presence of $100 \mathrm{mM}$ sodium azide and monitored by formation of the $\mathrm{Cu}$ (II) azido complex at $400 \mathrm{~nm}$. The reaction conditions differ from those reported in Fig. 6 (main manuscript) and Fig. S4 (SI) in that azide is present throughout the complete time course, rather than being added to aliquots after sampling. First order rate constants are as follows: $\mathrm{M} 49 \mathrm{H}$ (top traces) $6.3 \times 10^{-5} \mathrm{~s}^{-1}$, SeM-M49H (middle) $9.5 \times 10^{-5} \mathrm{~s}^{-1}$, and M47HM49H (3His) (bottom) $9.0 \times 10^{-5} \mathrm{~s}^{-1}$. Comparison with data in Table 3 (main manuscript) shows little effect for the $\mathrm{M} 49 \mathrm{H}$, and a percent decrease of 33 and 30 for the SeM and 3 His variants respectively. 

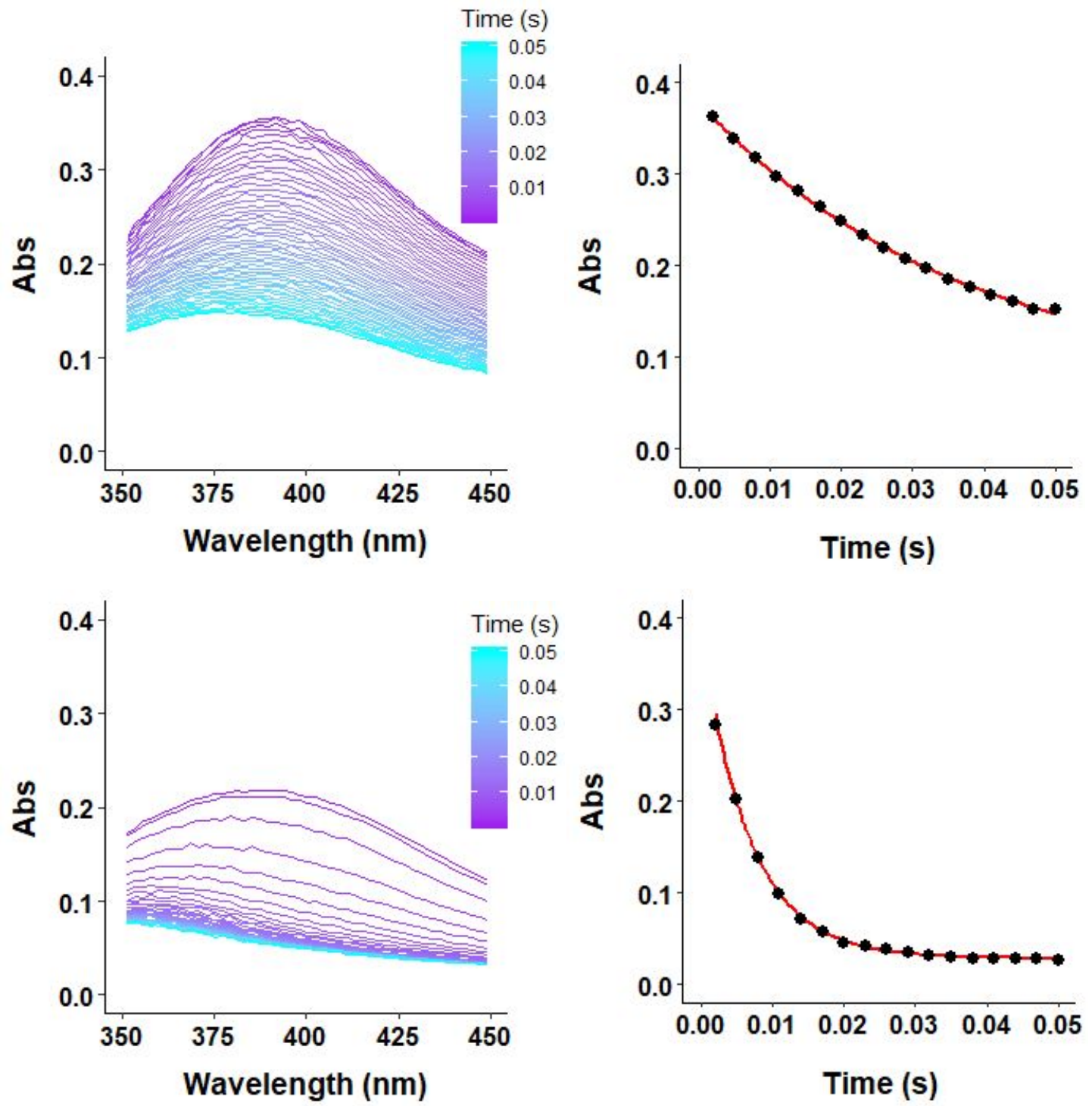

Figure S8. Stopped-flow measurements of the reduction of oxidized CusF M-site model variants with a $5 \mathrm{X}$ excess of buffered ascorbate. The resulting absorbance at $400 \mathrm{~nm}$ was plotted versus time fit to a single exponential decay with rate constants as listed in Table 3 of the main manuscript. Top traces are for the SeM-M49H and bottom traces are for the (M47HM49H) 3His. 
Table S1. Fits obtained to the Cu K EXAFS of the $\mathrm{Cu}(\mathrm{I})$ and $\mathrm{Cu}(\mathrm{II})-\mathrm{M} 47 \mathrm{HM} 49 \mathrm{H}$ (3His) derivatives of the CusF M-site model.

\begin{tabular}{|c|c|c|c|c|c|c|}
\hline Sample & Shell & $\mathrm{Fa}^{\mathrm{a}}$ & $\mathrm{No}^{\mathrm{b}}$ & $R(\AA ̊)^{c}$ & $\mathrm{DW}\left(\AA^{2}\right)^{\mathrm{d}}$ & $-\Delta \mathrm{E}_{0}$ \\
\hline $\mathrm{Cu}(\mathrm{I})-\mathrm{M} 47 \mathrm{HM} 49 \mathrm{H}$ & $\mathrm{Cu}-\mathrm{N}$ (His) & 0.47 & 3 & $1.948(3)$ & 0.011 & 1.75 \\
\hline \multirow[t]{4}{*}{$\mathrm{Cu}(\mathrm{I})-\mathrm{M} 47 \mathrm{HM} 49 \mathrm{H}-\mathrm{CO}$} & $\mathrm{Cu}-\mathrm{N}$ (His) & 0.46 & 3 & $2.008(3)$ & 0.009 & 1.76 \\
\hline & $\mathrm{Cu}-\mathrm{C}(\mathrm{CO})$ & & 1 & $1.804(6)$ & 0.002 & \\
\hline & $\mathrm{Cu}-\mathrm{O}(\mathrm{CO})$ & & 1 & $2.83(1)$ & 0.033 & \\
\hline & $\angle \mathrm{Cu}-\mathrm{C}-\mathrm{O}=178(11)^{\circ}$ & & & & & \\
\hline \multirow[t]{2}{*}{$\mathrm{Cu}(\mathrm{II})-\mathrm{M} 47 \mathrm{HM} 49 \mathrm{H}$} & $\mathrm{Cu}-\mathrm{N}(\mathrm{His})^{\mathrm{e}}$ & 0.61 & 3 & $1.993(4)$ & 0.010 & 4.9 \\
\hline & $\mathrm{Cu}-\mathrm{N} / \mathrm{O}^{f}$ & & 1 & $1.993(4)$ & 0.010 & \\
\hline \multirow[t]{2}{*}{$\mathrm{Cu}(\mathrm{II})-\mathrm{M} 47 \mathrm{HM} 49 \mathrm{H}-\mathrm{N}_{3}^{-}$} & $\mathrm{Cu}-\mathrm{N}(\mathrm{His})^{\mathrm{e}}$ & 0.80 & 3 & $2.000(4)$ & 0.009 & 4.0 \\
\hline & $\mathrm{Cu}-\mathrm{N} / \mathrm{O}^{\mathrm{f}}$ & & 1 & $2.000(4)$ & 0.009 & \\
\hline
\end{tabular}

a $\quad \mathrm{F}$ is a least-squares fitting parameter defined as $F^{2}=\frac{1}{N} \sum_{i=1}^{N} k^{6}(\text { Data }- \text { Model })^{2}$ where $\mathrm{N}$ is the number of data points and $k$ is the photoelectron wave vector defined as $k=2 \pi / h J\left(2 m_{e}\left(E-E_{0}\right)\right)$

b Coordination numbers are generally considered accurate to $\pm 25 \%$.

c Errors in bond lengths are reported as $95 \%$ confidence limits as determined from the least squares analysis. This underestimates the true error in the distances due to experimental factors such as finite data range, errors in the phase shifts, and choice of $\Delta \mathrm{E}_{0}$ which are strongly correlated with $\mathrm{R}$. True errors are probably closer to $0.02 \AA$ for first-shell (coordinated) ligands and $0.05 \AA$ for outer-shell (noncoordinated) ligands.

d Debye-Waller terms (DW) are calculated as exp $\left(-2 \sigma^{2} k^{2}\right)$ and reported as values of $2 \sigma^{2}\left(\AA^{2}\right)$.

e Fits included both single and multiple scattering contributions from the imidazole ring.

f In cases where the resolution $\Delta \mathrm{R}$ for split histidine and non-histidine shells is less than the theoretical resolution of the data $(\pi / 2 \mathrm{k})$, histidine and non-histidine scatterers are simulated as a single shell. 